Canad. Math. Bull. Vol. 22 (3), 1979

\title{
FUNCTIONAL DEPENDENCE AND ANALYTIC FUNCTIONS
}

\author{
BY \\ P. RAMANKUTTY
}

\begin{abstract}
Without appealing to the Cauchy theorem or its corollaries, it is proved that the real and imaginary parts of a non-constant complex-valued analytic function of several complex variables are functionally independent. This unifies and generalizes some results sporadically treated in standard treatises on function theory.
\end{abstract}

1. Introduction. The classical Liouville's theorem in complex function theory may be regarded as giving an estimate of the growth property of entire functions or as giving a criterion to decide if an analytic function defined on the whole complex plane is a trivial (i.e. constant) function or not. Either way, it does not give this sort of information about or test that could be applied to functions with domains smaller than the entire plane. The question naturally arises if there are indeed simple (i.e. not based on the Cauchy theorem or its consequences) criteria that could be applied to analytic functions defined on less extensive domains (i.e. connected open subsets) of the complex plane. Partial answers to this question appear to lie scattered in books on complex analysis where some sufficient simple criteria are given. (See for example: [1] Theorem 11 page 72, Problem 3 page 73; [3] Problem 9 page 50; [4] Problem 17 page 55 and [5] Exercises 7.2, 7.5 pages 86-87.) Occasionally, very essential use is also made of some of these results in establishing some rather important basic results such as the maximum modulus principle of function theory. (See for example [3] and [4]).

This note seeks to formulate a simple criterion which unifies the results mentioned above from [1], [3], [4] and indicates a manner of independence between the real and imaginary parts of a non-constant analytic function of one or several complex variables. In order to make the note somewhat selfcontained, the notion of functional dependence is defined first and a basic theorem involving this notion is quoted (Theorem 2.2). The main result of this note (Theorem 3.1) is introduced next. It may be noted that the one-variable case of this theorem is in itself a generalization of the results referred to above from [1], [3], [4] and does, indeed, unify them. Although this result is an obvious consequence of the open mapping theorem, the proof presented here is not based on that theorem or on any other property of analytic functions

Received by the editors June 27, 1978. 
derived from the Cauchy theorem. That the functional independence of the components of a function $F: \mathbb{R}^{n} \rightarrow \mathbb{R}^{m}$ does not imply that $F$ is an open map (although the converse is trivially true) is demonstrated by the easy example in 3.2. That the result of theorem 3.1 does not, as such, hold for vector-valued analytic functions is demonstrated by the elementary example in 3.3 where a much stronger type of functional dependence is exhibited between the real and imaginary parts of a non-constant analytic function taking values in $\mathbb{C}^{2}$.

\section{Functional dependence.}

2.1 Definttion. Let $\Omega$ be an open set in $\mathbb{R}^{n}$ and $f_{1}, \ldots, f_{m}$ be real-valued functions defined on $\Omega$. Then $f_{1}, \ldots, f_{m}$ are said to be functionally dependent over $\Omega$ if and only if there exists a function $\varphi: \mathbb{R}^{m} \rightarrow \mathbb{R}$ such that $\varphi^{-1}(0)$ has empty interior and $\varphi\left(f_{1}(x), \ldots, f_{m}(x)\right)=0$ for all $x$ in $\Omega$.

The following theorem regarding functional dependence is only a slight modification of theorem 7.61 of [2]; its proof is, therefore, omitted.

2.2 THEOREM. Let $\Omega$ be an open set in $\mathbb{R}^{n}$ and $f: \Omega \rightarrow \mathbb{R}^{m}$ be continuously differentiable. If there exists a point $c \in \Omega$ such that the linear map $f^{\prime}(c): \mathbb{R}^{n} \rightarrow \mathbb{R}^{m}$ is surjective, then the $m$ components of $f$ are functionally independent over $\Omega$.

3. Application to analytic functions. The principal result of this note regarding the functional independence of the real and imaginary parts of an analytic function of several complex variables may now be established.

3.1 Theorem. Let $\Omega$ be a domain in $\mathbb{R}^{2 n}$, $G$ be the domain in $\mathbb{C}^{n}$ defined by $G=\left\{\left(x_{1}+i y_{1}, \ldots, x_{n}+i y_{n}\right) \in \mathbb{C}^{n}:\left(x_{1}, y_{1}, \ldots, x_{n}, y_{n}\right) \in \Omega\right\}$ and let $f: G \rightarrow \mathbb{C}$ be an analytic function. Then $\operatorname{Re} f$ and $\operatorname{Im} f$ are functionally dependent over $\Omega$ if and only if $f$ is constant.

Proof. Since the "if" part is obvious, suppose that $f$ is not constant. Then there exists a point $c=\left(a_{1}, b_{1}, \ldots, a_{n}, b_{n}\right) \in \Omega$ with $f^{\prime}(a+i b) \neq 0$ where $a=$ $\left(a_{1}, \ldots, a_{n}\right)$ and $b=\left(b_{1}, \ldots, b_{n}\right)$. If $u=\operatorname{Re} f$ and $v=\operatorname{Im} f$, then by the CauchyRiemann equations,

$$
f^{\prime}=\left(\frac{\partial u}{\partial x_{1}}+i \frac{\partial v}{\partial x_{1}}, \ldots, \frac{\partial u}{\partial x_{n}}+i \frac{\partial v}{\partial x_{n}}\right)
$$

so that

$$
0<\left\|f^{\prime}(a+i b)\right\|^{2}=\sum_{k=1}^{n}\left(\frac{\partial u(c)}{\partial x_{k}}\right)^{2}+\left(\frac{\partial v(c)}{\partial x_{k}}\right)^{2} .
$$

Hence $\left(\frac{\partial u(c)}{\partial x_{k}}\right)^{2}+\left(\frac{\partial v(c)}{\partial x_{k}}\right)^{2}>0$ for some $k(1 \leq k \leq n)$. Using the Cauchy- 
Riemann equations again, this condition may be written

$$
\operatorname{det}\left(\begin{array}{ll}
\frac{\partial u}{\partial x_{k}}(c) & \frac{\partial u}{\partial y_{k}}(c) \\
\frac{\partial v}{\partial x_{k}}(c) & \frac{\partial v}{\partial y_{k}}(c)
\end{array}\right)>0
$$

This shows that at the point $c \in \Omega$, the function $g: \Omega \rightarrow \mathbb{R}^{2}$ defined by $g=(u, v)$ has a derivative $g^{\prime}(c)$ which maps $\mathbb{R}^{2 n}$ onto $\mathbb{R}^{2}$. It follows from theorem 2.2 that $u, v$ are functionally independent over $\Omega$.

3.2 ExAmple. The range of the function $F: \mathbb{R}^{2} \rightarrow \mathbb{R}^{2}$ defined by $F(x, y)=$ $\left(x^{2}+y^{2}, 2 x y\right)$ is $\left\{(x, y) \in \mathbb{R}^{2}:|y| \leq x\right\}$; so $F$ is not an open map.

3.3 ExAmple. Let $G$ be any domain in $\mathbb{C}^{n}, f: G \rightarrow \mathbb{C}$ be any non-constant analytic function and $F: G \rightarrow \mathbb{C}^{2}$ be defined by $F=(f,-f)$. Then writing $F=$ $\left(F_{1}, F_{2}\right)$ and $u_{k}=\operatorname{Re} F_{k}$ and $v_{k}=\operatorname{Im} F_{k}$, we have $u_{2}=-u_{1}, v_{2}=-v_{1}$ so that $u_{1}, u_{2}, v_{1}, v_{2}$ satisfy the functional relation $\varphi\left(u_{1}, u_{2}, v_{1}, v_{2}\right)=0$ where $\varphi: \mathbb{R}^{4} \rightarrow \mathbb{R}$ is defined by

$$
\begin{aligned}
\varphi\left(x_{1}, x_{2}, x_{3}, x_{4}\right)= & A\left(x_{1}, x_{2}, x_{3}, x_{4}\right)\left(g\left(x_{1}\right)-g\left(-x_{2}\right)\right) \\
& +B\left(x_{1}, x_{2}, x_{3}, x_{4}\right)\left(h\left(x_{3}\right)-h\left(-x_{4}\right)\right),
\end{aligned}
$$

$A, B$ being any two real-valued functions defined on $\mathbb{R}^{4}$ and $g, h$ being any two real-valued functions defined on $\mathbb{R}$. Since $A, B, g, h$ can be taken arbitrarily smooth and without singularities, it is obvious that the function $\varphi$ can also be chosen to be arbitrarily smooth and without singularities; nevertheless, $F$ is non-constant.

\section{REFERENCES}

1. L. V. Ahlfors, Complex Analysis, 2nd Edition, McGraw-Hill, New York, 1966. MR 32 \#5844.

2. J. C. Burkill and H. Burkill, A Second Course in Mathematical Analysis, Cambridge Univ. Press, 1970. MR 41 \#3197.

3. R. V. Churchill, J. W. Brown and R. F. Verhey, Complex Variables and Applications, 3rd Edition, McGraw-Hill, New York, 1974. MR 22 \#3793 and MR 50 \#580.

4. J. B. Conway, Functions of One Complex Variable, Springer-Verlag, New York, 1973.

5. M. Heins, Complex Function Theory, Academic Press, New York, 1968. MR 39 \#413.

DEPARTMENT OF MATHEMATICS

UNIVERSITY OF AUCKLAND

AUCKLAND, New ZEALAND 